\title{
A Constrained Coding Approach to Error-Free Half-Duplex Relay Networks
}

\author{
Frank R. Kschischang Fellow, IEEE and Tobias Lutz
}

\begin{abstract}
We show that the broadcast capacity of an infinite-depth tree-structured network of error-free half-duplexconstrained relays can be achieved using constrained coding at the source and symbol forwarding at the relays.
\end{abstract}

Index Terms-Relay channels, constrained coding, half-duplex constraint.

\section{INTRODUCTION}

$\mathbf{I}$ NFORMATION transmission through a relay channel or network with error-free and/or half-duplex-constrained relays is a problem that has been considered by several authors [1]-[7]. In this paper the focus is on directed trees of errorfree half-duplex-constrained relays, as shown in Fig. 1. Such networks include a chain of relays as a special case. The transmission objective is to broadcast information from a source (situated at the root of the tree) to all network nodes, each of which is half-duplex constrained. In each time slot, a node either receives (without error) the transmission of its parent, or broadcasts information to its children, but it may not do both.

More precisely, we assume that transmission between nodes in the network occurs in discrete time-slots. Let $\mathcal{Q}:=$ $\{0, \ldots, q-1\}$ be a $q$-ary transmission alphabet, let $\mathrm{N}$ be an additional symbol which indicates a channel use without transmission, and let $\mathcal{X}:=\mathcal{Q} \cup\{\mathrm{N}\}$. In any given time-slot, each node of the network broadcasts a symbol $x \in \mathcal{X}$ to its children; the node is said to be oN if $x \in \mathcal{Q}$; otherwise $x=\mathrm{N}$ and the node is said to be OFF.

The half-duplex constraint is captured as follows. When a relay is OFF, it is connected to its parent through a noiseless $(q+1)$-ary channel with alphabet $\mathcal{X}$, and so receives the transmission from its parent without error. When a relay is $\mathrm{ON}$, it cannot receive, so the symbol sent by its parent is erased.

The simplest approach to information broadcasting is to require each network node to be OFF half of the time, organized in deterministic fashion so that a node is OFF whenever its parent might be ON. Nodes simply forward what they receive, resulting in a transmission rate of $0.5 \log _{2}(q+1)$ bits per

Submitted for publication on December 13, 2011; revised November 17, 2012 and May 28, 2013.

F. R. Kschischang is with the Electrical and Computer Engineering Department, University of Toronto, 10 King's College Road, Toronto, Ontario M5S 3G4, Canada (e-mail: frank@comm.utoronto.ca), Tobias Lutz is with the Lehrstuhl für Nachrichtentechnik, Technische Universität München, D80290 München, Germany (e-mail: tobi.lutz@tum.de). The work of Frank R. Kschischang was supported by a Hans Fischer Senior Fellowship from the Technische Universität München, Institute for Advanced Study, funded by the German Excellence Initiative.

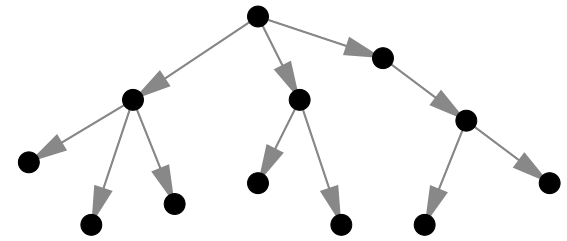

Fig. 1. A broadcast tree of depth $D=3$.

symbol (b/sym). The broadcast capacity, on the other hand, approaches [7]

$$
C(q):=\log _{2}\left(\frac{1+\sqrt{4 q+1}}{2}\right) \text { b/sym }
$$

as the tree-depth becomes large. In the binary case, deterministic store-and-forward achieves $0.5 \mathrm{~b} / \mathrm{sym}$ whereas $C(1)=$ $\log _{2} \phi=0.6924$, where $\phi$ is the golden ratio. For trees of finite depth, even greater rates are possible. For example, for trees of depth $D=2$, a rate of $0.7729 \mathrm{~b} / \mathrm{sym}$ is achievable in the binary case. It is clear that deterministic store-andforward falls short of the maximum possible transmission rate. However, to achieve the broadcast capacity of trees of finite depth requires a sophisticated coding approach based on coding additional information in the ON-OFF patterns of the nodes [7].

We note that ON-OFF patterns have also been exploited for neighbor discovery in half-duplex-constrained networks using a compressed sensing approach [8]-[10]. Another problem, namely a line of three nodes where the first two nodes are half-duplex sources and where all nodes are connected by packet erasure channels, was addressed in [11] within a queuing-theoretic framework. In [12] a Gaussian point-topoint channel with a sender subject to a duty cycle constraint (e.g., a half-duplex constraint) and an average power constraint is considered. Interestingly, the optimal input distribution is shown to be discrete, i.e., a modulated ON-OFF signaling scheme is capacity-achieving.

In this paper we will present a broadcasting scheme, based on constrained coding, that preserves the simplicity of the store-and-forward approach, but achieves a higher transmission rate than deterministic store-and-forward. In particular, we show that we can achieve a broadcast rate of $C(q)$ in any error-free half-duplex-constrained tree network using constrained coding at the source and symbol forwarding at the relays. 


\section{Constrained Coding BACKGRound}

The approach we take to broadcasting in a tree of halfduplex-constrained nodes uses tools from constrained coding (or symbolic dynamics); see, e.g., [13], [14]. In a nutshell, the field of constrained coding studies mappings from unconstrained input sequences to output sequences obeying certain constraints. The constraints are often expressed by specifying forbidden sub-blocks, i.e., subsequences that are not permitted to occur in any output sequence. A classical example is the golden mean shift, which is the set of binary sequences in which the sub-block 11 never occurs. Constrained coding has found many applications in magnetic and optical recording systems.

The capacity of a constrained system, which is the maximum rate at which unconstrained binary data may be mapped to constrained output data, is defined as

$$
C=\limsup _{n \rightarrow \infty} \frac{1}{n} \log _{2} N(n) \text { b/sym, }
$$

where $N(n)$ denotes the number of sequences in the output alphabet having length $n$ and satisfying the given constraint. For example, the golden mean shift satisfies the Fibonacci recurrence: for $n \geq 2$,

$N(n)=N(n-1)+N(n-2)$, with $N(0)=1, N(1)=2$.

From this it can be shown that the golden mean shift has $C=\log _{2} \phi$, where $\phi=(1+\sqrt{5}) / 2$ is the golden ratio (a result that explains the name "golden mean shift"). Interestingly, the golden ratio also arises in the analysis of the trapdoor channel [15] with feedback, where it is shown that the capacity equals $\log _{2} \phi$.

It is well known that the capacity of certain constrained systems can be obtained via an irreducible, lossless graph presentation of the constraint [13]. If $G$ is such a presentation, and $A_{G}$ is the adjacency matrix of $G$, then

$$
C=\log _{2} \lambda\left(A_{G}\right)
$$

where $\lambda$ is the largest of the absolute values of the eigenvalues of $A_{G}$. This formulation of capacity will be used in the sequel.

\section{Code Construction}

We now describe the constrained coding approach taken in this paper. The transmission protocol is trivial, amounting to simple symbol-forwarding: during any given time-slot, every non-source node simply forwards (to all of its children) the symbol it has received from its parent during the previous time-slot. Correct forwarding is achieved provided that nodes obey the half-duplex constraint, i.e., that they are never ON when their parent node might be ON. Under the symbolforwarding protocol, this is accomplished if and only if the source is never itself ON in two adjacent time-slots.

Thus we arrive naturally at a constrained coding problem: the source may emit any sequence of symbols drawn from $\mathcal{X}$ satisfying the constraint that no two adjacent symbols are drawn from $\mathcal{Q}$. In the language of symbolic dynamics, every transmitted sequence is drawn from the shift of finite type denoted as $\mathcal{X}_{\mathcal{Q}^{2}}$ having forbidden sub-block set $\mathcal{Q}^{2}:=\mathcal{Q} \times$
$\mathcal{Q}$. An irreducible, lossless graph presentation of this shift is shown in Fig. 2. When $q=1$, this shift is equivalent to the golden mean shift.

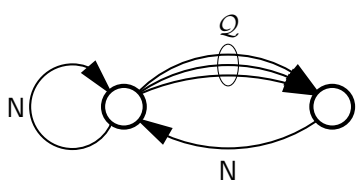

Fig. 2. Graph presentation of half-duplex constraint under symbolforwarding.

The adjacency matrix of this presentation is given, as a function of $q$, as

$$
A(q)=\left[\begin{array}{ll}
1 & q \\
1 & 0
\end{array}\right]
$$

which has characteristic polynomial

$$
p_{q}(\lambda)=\lambda^{2}-\lambda-q .
$$

The eigenvalues of $A(q)$ (the roots of $p_{q}(\lambda)$ ) are given as

$$
\lambda=\frac{1}{2}(1 \pm \sqrt{1+4 q}),
$$

and the constrained capacity (the logarithm of the largest eigenvalue) is given as

$$
C(q)=\log _{2}\left(\frac{1+\sqrt{1+4 q}}{2}\right) .
$$

Remarkably - and this is the central result of this paper-the constrained coding approach achieves the broadcast capacity of infinite-depth trees, but without the necessity of designing sophisticated timing codes as in [7].

The capacity $C(q)$ can be approached using methods (e.g., the state-splitting algorithm) from constrained coding. Fig. 3 provides two examples. The first, in Fig. 3(a), is a standard example in constrained coding [14] and gives a rate-(2/3) encoder for $q=1$, which achieves more than $96 \%$ of the capacity $C(1)=\log _{2}(\phi)$. The second, in Fig. 3 b), is a rate(3/2) encoder for $q=6$, which achieves more than $94 \%$ of the capacity $C(6)=\log _{2}(3)$. The encoder can be constructed in three steps. First, the second power graph is generated from the golden mean shift shown in Fig 2. Subsequently, the state with most outgoing edges is split (according to the splitting criteria of the state-splitting algorithm). In the final step, sufficiently many edges are deleted so that eight outgoing edges per state remain. Similar examples can readily be constructed for other values of $q$. For any given $q$, if the number of encoder states is allowed to grow, $C(q)$ can be approached arbitrarily closely.

\section{Discussion}

Table I compares, for $q=1$, rates achievable in networks of finite depth $D$ using three approaches: the timing codes presented in [7], the constrained coding approach of this paper, and the deterministic store-and-forward approach. The row labelled $C$, which gives the maximum achievable rate (using timing codes), serves as a benchmark for the other schemes. We observe that $C(q) / C$, the relative efficiency of constrained 


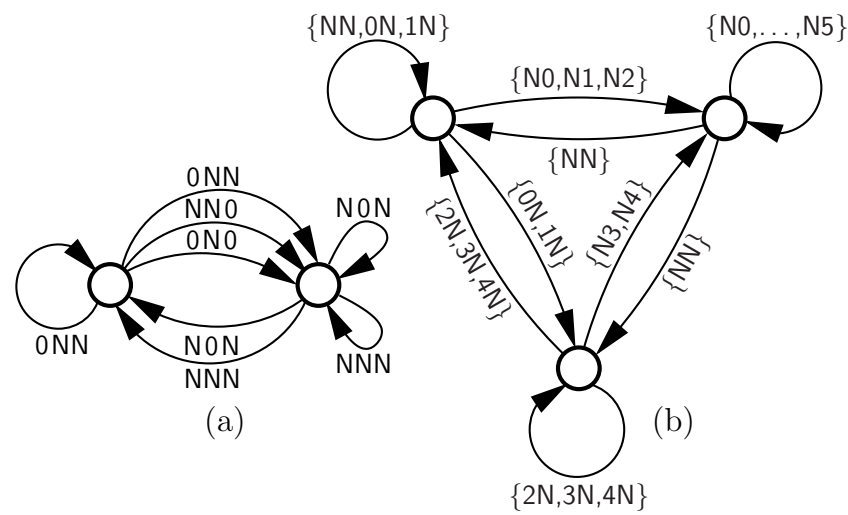

Fig. 3. Encoders for (a) $q=1, R=2 / 3, C_{1}=\log _{2} \phi \approx 0.6942 \mathrm{~b} / \mathrm{sym}$, (b) $q=6, R=3 / 2, C_{6}=\log _{2} 3 \approx 1.5850 \mathrm{~b} / \mathrm{sym}$.

TABLE I

Achievable Rates in Networks of Finite Depth $D$ with $q=1$

\begin{tabular}{r|ccccc}
$D$ & 2 & 3 & 5 & 11 & $\infty$ \\
\hline \hline$C$ (b/sym) & 0.7729 & 0.7324 & 0.7099 & 0.6981 & 0.6942 \\
$C(q) / C(\%)$ & 89.82 & 94.79 & 97.80 & 99.44 & 100 \\
$0.5 / C(\%)$ & 64.70 & 68.27 & 70.43 & 71.62 & 72.02
\end{tabular}

coding, rapidly converges to unity as $D$ increases. On the other hand, $0.5 / C$, the relative efficiency of deterministic storeand-forward, saturates at approximately $72 \%$. The differences among the three approaches however become smaller as $q$ increases.

We observe that timing codes require nodes at different depths in a finite tree to have different (carefully designed) ON-OFF duty cycles that depend on the depth of the tree. It is shown in [7] that these duty cycles converge to a constant as the tree depth grows. The capacity $C(q)$, achieved both by timing codes and by the constrained coding approach of this paper, is the maximum rate that can be achieved with a constant ON-OFF duty cycle throughout the network.

As a final remark, we note that it might be interesting to consider a network model with noisy transmission links. In this case, techniques that combine constrained coding with error control (as in, e.g., [16]-[18]) may be helpful.

\section{REFERENCES}

[1] P. Vanroose and E. C. van der Meulen, "Uniquely decodable codes for deterministic relay channels," IEEE Trans. Info. Theory, vol. 38, no. 4, pp. 1203-1212, Jul. 1992

[2] G. Kramer, "Models and Theory for Relay Channels with Receive Constraints," in Proc. 42nd Annual Allerton on Conf. Commun., Control, and Computing, Monticello, IL, Sep. 29-Oct. 12004.

[3] _ - "Communication strategies and coding for relaying," in Wireless Communications, ser. IMA Volumes in Mathematics and its Applications. New York: Springer, 2007, vol. 143, pp. 163-175.

[4] S. Vijayakumaran, T. F. Wong, and T. M. Lok, "Capacity of the degraded half-duplex relay channel," 2007, available online: http://arxiv.org/abs/0708.2270

[5] T. Lutz, G. Kramer, and C. Hausl, "Capacity for half-duplex line networks with two sources," in Proc. IEEE Int. Symp. Info. Theory, Austin, TX, Jun. 13-18 2010, pp. 2393-2397.

[6] P. Popovski and O. Simeone, "Protocol coding for two-way communications with half-duplex constraints." in Proc. IEEE Global Commun. Conf. (GLOBECOM), Miami, FL, Dec. 6-Dec. 10 2010, pp. 1-5.

[7] T. Lutz, C. Hausl, and R. Kötter, "Bits through deterministic relay cascades with half-duplex constraint," IEEE Trans. Info. Theory, vol. 58, no. 1, pp. 369-381, Jan. 2012.
[8] L. Zhang, J. Luo, and D. Guo, "Neighbor discovery for wireless networks via compressed sensing," Perf. Eval., vol. 70, pp. 457-471, 2013.

[9] D. Guo and L. Zhang, "Virtual full-duplex wireless communication via rapid on-off-division duplex," in Proc. 48th Annual Allerton on Conf. Commun., Control, and Computing, Monticello, IL, Sep. 29-Oct. 1 2010.

[10] L. Applebaum, W. U. Bajwa, R. Calderbank, and S. Howard, "Choir codes: Coding for full duplex interference management," in Proc. 49th Annual Allerton Conf. on Commun., Control, and Computing, Monticello, IL, Sep. 28-30 2011.

[11] D. E. Lucani and J. Kliewer, "Energy-delay considerations in coded packet flows," in Proc. IEEE Int. Symp. Info. Theory, St. Petersburg, Russia, Jul. 31-Aug. 5 2011, pp. 796-800.

[12] L. Zhang and D. Guo, "Capacity of Gaussian channels with duty cycle and power constraints," in Proc. IEEE Int. Symp. Info. Theory, St. Petersburg, Russia, Jul. 31-Aug. 5 2011, pp. 513-517.

[13] D. A. Lind and B. H. Marcus, An Introduction to Symbolic Dynamics and Coding. Cambridge, 1995.

[14] B. H. Marcus, R. M. Roth, and P. H. Siegel, "An introduction to coding for constrained systems," 2001, available online: http://www.math.ubc.ca/ marcus/Handbook

[15] H. Permuter, P. Cuff, B. VanRoy, and T. Weissman, "Capacity of the trapdoor channel with feedback," IEEE Trans. Info. Theory, vol. 54, no. 7, pp. 3150-3165, Jul. 2008

[16] W. G. Bliss, "Circuitry for performing error correction calculations on baseband encoded data to eliminate error propagation," IBM Tech. Discl. Bull., vol. 23, pp. 4633-4634, Mar. 1981.

[17] A. J. van Wijngaarden and K. A. S. Immink, "Maximum runlengthlimited codes with error control capabilities," IEEE J. Select. Areas Commun., vol. 19, no. 4, pp. 602-611, Apr. 2001.

[18] H. Y. Chen, M. C. Lin, and Y. L. Ueng, "Low-density parity-check coded recording systems with run-length-limited constraints," IEEE Trans. Magnetics, vol. 44, no. 9, pp. 2235-2242, Sep. 2008.

Frank R. Kschischang received the B.A.Sc. degree (with honors) from the University of British Columbia in 1985 and the M.A.Sc. and Ph.D. degrees from the University of Toronto in 1988 and 1991, respectively, all in electrical engineering. $\mathrm{He}$ is a Professor and Canada Research Chair at the University of Toronto, where he has been a faculty member since 1991. Between 2011 and 2013 he was a Hans Fischer Senior Fellow at the Institute for Advanced Study, Technische Universität München.

His research interests are focused primarily on the area of channel coding techniques, applied to wireline, wireless and optical communication systems and networks. He is the recipient of the 2010 Killam Research Fellowship, the 2010 Communications Society and Information Theory Society Joint Paper Award and the 2012 Canadian Award in Telecommunications Research. He is a Fellow of IEEE, of the Engineering Institute of Canada, and of the Royal Society of Canada.

During 1997-2000, he served as an Associate Editor for Coding Theory for the IEEE TRANSACTIONS ON INFORMATION THEORY. He also served as technical program co-chair for the 2004 IEEE International Symposium on Information Theory (ISIT), Chicago, and as general co-chair for ISIT 2008, Toronto. He served as the 2010 President of the IEEE Information Theory Society.

Tobias Lutz was born in Krumbach, Germany, on May 2, 1980. He received the B.Sc. and Dipl.-Ing. degrees in electrical engineering and information technology from the Technische Universität München, Germany, in 2007 and 2008, respectively. Funded by a grant from the American European Engineering Exchange program (AE3), he studied electrical and computer engineering at the Rensselaer Polytechnic Institute, Troy, NY, during the academic year 2005/06. Since 2008, he has been with the Institute for Communications Engineering, Technische Universität München, where he is currently working toward the Dr.-Ing. degree under the supervision of Prof. Gerhard Kramer. Since 2009 he has been studying financial mathematics at the Ludwig Maximilian Universität München. Tobias Lutz was awarded a Qualcomm Innovation Fellowship in 2012. From August 2012 through March 2013 he was a visiting graduate student at Stanford University. His current research interests include information and coding theory and their application to wireless relay networks. 